\title{
Baicalein inhibits prostate cancer cell growth and metastasis via the caveolin-1/AKT/mTOR pathway
}

\author{
Zhaoxin Guo ${ }^{1}$ Xiaolin $\mathrm{Hu}^{2} \cdot$ Zhaoquan Xing ${ }^{1} \cdot \mathrm{Rui}_{\mathrm{Xing}}{ }^{3} \cdot$ Renguang $\mathrm{Lv}^{4}$.

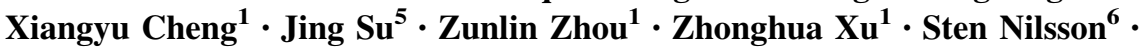 \\ Zhaoxu Liu ${ }^{1,5,6}$
}

Received: 27 January 2015/ Accepted: 30 April 2015/Published online: 10 May 2015

(C) The Author(s) 2015. This article is published with open access at Springerlink.com

\begin{abstract}
Prostate cancer (PCa) is lethal type of genitourinary cancer due to its high morbidity and gradual resistance to androgen deprivation therapy. Accumulating evidence has recently suggested that the daily intake of flavonoids is negatively correlated with the risk of cancer. In this study, we aimed to investigate the potential effects of baicalein on androgen-independent PCa cells and the underlying mechanisms through which baicalein exerts its actions. Cell viability and flow cytometric apoptosis assays indicated that baicalein potently suppressed the growth and induced the apoptosis of DU145 and PC-3 cells in a timeand dose-dependent manner. Consistently, the inhibitory effects of baicalein on migration and invasion were also observed in vitro. Mechanistically, we found that baicalein can suppress caveolin-1 and the phosphorylation of AKT and mTOR in a time- and dose-dependent manner. Moreover, the inhibition of the activation of AKT with LY294002 significantly promoted the apoptosis and metastasis induced by baicalein. In conclusion, these
\end{abstract}

Zhaoxu Liu

sdlzx2k@126.com; zhaoxvl@sdu.edu.cn

1 Department of Urology, Qilu Hospital of Shandong University, Jinan 250012, Shandong, China

2 Department of Endocrinology, Jinan Central Hospital, Shandong University, Jinan 250013, Shandong, China

3 Department of Urology, Linyi County People's Hospital, Dezhou 251500, Shandong, China

4 Department of Urology, Jinan Iron and Steel General Hospital, Jinan 250000, Shandong, China

5 School of Nursing, Shandong University, No. 107 Wenhua Xi Road, Jinan 250012, Shandong, China

6 Department of Oncology and Pathology, Karolinska Institutet, 17177 Stockholm, Sweden findings suggested that baicalein can induce apoptosis and inhibit metastasis of androgen-independent $\mathrm{PCa}$ cells through inhibition of the caveolin-1/AKT/mTOR pathway, which implies that baicalein may be a potential therapeutic agent for the treatment of androgen-independent prostate cancer patients.

Keywords Baicalein - Anti-cancer activity - Androgenindependent prostate cancer · Metastasis · Caveolin-1

\section{Introduction}

Prostate cancer $(\mathrm{PCa})$ is the most common malignant cancer in men and the second leading cause of cancerrelated death being only inferior to lung cancer [1]. At present, androgen deprivation therapy (ADT) is still the main treatment for PCa. However, within 2 years, the majority of prostate cancer becomes androgen independent and progresses into castration-resistant prostate cancer (CRPC) stage, at which point few drugs are effective, giving rise to high mortality $[2,3]$. Therefore, the development of pharmacological interventions to retard prostate tumor growth after the emergence of androgen-independent disease is urgently needed.

Flavonoids are widely used Chinese herbal medicines. Epidemiological studies now indicate that an increased intake of dietary flavonoids is associated with a decreased risk of some cancers $[4,5]$. Baicalein is a bioactive flavonoid derived from the root of Scutellaria baicalensis, and has been widely used to treat inflammation, cardiovascular diseases, and infections [6]. Baicalein alone, or in combination with other agents, can also inhibit cancer cell growth and metastasis in breast cancer, hepatocellular carcinoma, leukemia, and colon cancer [7-10]. In addition, 
Chen previously found that baicalein can regulate the expression of the androgen receptor, inhibit the proliferation of prostate cancer cells, and induce the apoptosis of these cells [11]. However, the effects of baicalein on the metastasis of androgen-independent prostate cancer cells and the underlying molecular mechanisms have not been well established.

In this study, the DU145 and PC-3 cell lines were selected as androgen-independent prostate cancer cells. Our data established the growth-inhibitory and pro-apoptotic effects of baicalein on PCa cells in vitro. Simultaneously, we found that baicalein can potently inhibit the migration and invasion of $\mathrm{PCa}$ cells. Further investigation of the underlying mechanisms indicated that the caveolin-1/AKT/ mTOR pathway plays key roles in these actions. The effects of baicalein on androgen-independent cells provide useful information for the treatment of CRPC.

\section{Materials and methods}

\section{Cell lines and reagents}

This study was approved by the Qilu Hospital Committee of Shan Dong University. Androgen-independent PCa cell lines (DU145 and PC-3) were purchased from the Type Culture Collection of the Chinese Academy of Sciences (Shanghai, China). All of the cells were grown in RPMI1640 medium (Hyclone, Utah, USA) containing $10 \%$ fetal calf serum (Gibco, CA, USA) at $37{ }^{\circ} \mathrm{C}$ in a $5 \% \mathrm{CO}_{2}$ incubator. Baicalein was purchased from Selleck Chemicals (Houston, TX, USA) and was dissolved according to the manufacturer' instructions. Antibodies against phosphoAKT (Ser473), AKT, phospho-mTOR, mTOR and GAPDH, and HRP-conjugated goat anti-rabbit and antimouse IgG antibodies were obtained from Cell Signaling Technology (Beverly, MA, USA). Antibodies against cleaved PARP, Bax, Bcl-2, and survivin were obtained from Abcam (Abcam, Cambridge, UK).

\section{Cell viability assay}

The cell viability was determined by the CCK8 assay. Briefly, DU145 or PC-3 $\left(4 \times 10^{3}\right.$ cells/well $)$ cells in $200 \mu \mathrm{l}$ of medium were seeded into 96-well plates. After $12 \mathrm{~h}$ of incubation to allow adherence, the medium was replaced with medium containing different concentrations of baicalein and the plate was incubated for 24,48 , and $72 \mathrm{~h}$. The cultured cells were subsequently treated with $20 \mu$ of Cell Counting kit-8 (Dojindo, Japan) and incubated at $37{ }^{\circ} \mathrm{C}$ for an additional $4 \mathrm{~h}$ according to the manufacturer's instructions. The absorbance values were determined at $450 \mathrm{~nm}$ using a microplate reader (Bio-Rad, Hercules, CA, USA).

\section{In vitro scratch assay}

The PCa cells were seeded in 24-well plates. After incubation for $24 \mathrm{~h}$, each well was manually scratched with a $200 \mu 1$ pipette tip, washed three times with PBS, and incubated with baicalein ( 20 and $40 \mu \mathrm{M}$, respectively) at $37^{\circ} \mathrm{C}$. Images were obtained again after $24 \mathrm{~h}$ of incubation. The distance between two cell edges was analyzed using the ImageJ software.

\section{Transwell invasion and migration assay}

The Transwell invasion assay was performed as described previously [12]. Briefly, a total of $4 \times 10^{4}$ cells suspended in $100 \mu \mathrm{l}$ of serum-free medium were added to the upper chambers of the Transwell system (24 wells, $8-\mathrm{mm}$ pore size; Corning Costar, Lowell, MA, USA) coated with $2 \mathrm{mg} / \mathrm{ml}$ Matrigel (BD Biosciences). RPMI-1640 containing $20 \%$ FBS and baicalein $(20$ and $40 \mu \mathrm{M})$ was then added to the lower chamber. After $24 \mathrm{~h}$, the non-invaded cells in the upper chamber were gently removed with a cotton swab, and the cells attached to the lower surface were fixed with precooled methanol and stained with $0.1 \%$ crystal violet. Five fields of each chamber were randomly selected, and the cell numbers were counted under a microscope.

For the migration assay, the cells were seeded into upper chambers that were not coated with Matrigel. The following steps in the assay were the same in the invasion assay. After $24 \mathrm{~h}$, the cells on five randomly selected fields in the lower surface were counted, and the cell numbers were then subjected to statistical analysis.

\section{Apoptosis assay}

Cell apoptosis was detected using the Annexin V-FITC/PI apoptosis detection kit (BD Biosciences, San Jose, CA, USA). In brief, $1 \times 10^{5}$ untreated or baicalein-treated cells were harvested following trypsinization and centrifugation. The cells were washed with PBS and resuspended in $500 \mu \mathrm{l}$ of binding buffer. After $5 \mu \mathrm{l}$ of Annexin V-FITC was added, the cells were incubated after $5 \mathrm{~min} 5 \mu \mathrm{l}$ of PI (propidium iodide) was added, and the cells were incubated 15 min in the dark. The data were analyzed using the WinMDI V2.9 software (The Scripps Research Institute, San Diego, CA, USA).

\section{Western blotting}

The cells were lysed in RIPA buffer containing $1 \%$ protease inhibitors. Equal amounts of proteins from each sample were separated by SDS-PAGE and transferred onto a PVDF membrane using a wet transfer apparatus (Bio- 
Rad, Hercules, CA, USA). The membranes were blocked with $5 \%$ non-fat milk for $2 \mathrm{~h}$ at room temperature and incubated with the primary antibodies overnight at $4{ }^{\circ} \mathrm{C}$. The membranes were then exposed to the horseradish peroxidase-labeled secondary antibodies for $1 \mathrm{~h}$ at room temperature and detected using an enhanced chemiluminescence detection system (Amersham, Pittsburg, PA, USA). The protein levels were analyzed using the ImageJ software.

\section{Statistical analysis}

The data are mostly presented as the means \pm SD. The SPSS software package (version 13.0; SPSS, Chicago, IL, USA) was used for all of the statistical analyses. Significant differences between the treatment and control values were analyzed by Student's two-tailed $t$ test or one-way analysis of variance wherever appropriate. Differences were considered statistically significant if $P<0.05$. Each variable was tested twice and the experiments were repeated three times.

\section{Results}

\section{Baicalein inhibits proliferation of prostate cancer cells}

To study the effects of baicalein on the proliferation of $\mathrm{PCa}$ cells, DU145 and PC-3 cells were exposed to different concentrations of baicalein for 24,48 , and $72 \mathrm{~h}$, and their

A<smiles>O=c1cc(-c2ccccc2)oc2cc(O)c(O)c(O)c12</smiles>

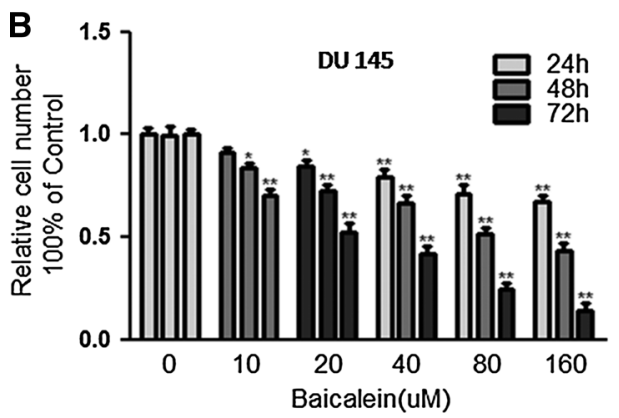

Fig. 1 Baicalein suppresses the viability of DU 145 and PC-3 cells. a Structure of baicalein. The effect of baicalein on cell viability was measured by the CCK-8 assay. b DU145 cells and c PC-3 cells were treated with different concentrations of baicalein for 24,48 , and $72 \mathrm{~h}$. proliferation was analyzed using the CCK-8 assay. The results showed that baicalein significantly inhibited the proliferation of DU145 and PC-3 cells in a time- and dosedependent manner $(* P<0.05, * * P<0.01)$ (Fig. 1b, c). The viability of DU145 and PC-3 cells was reduced to 58.4 and $52.7 \%$ after treatment with baicalein $(40 \mu \mathrm{M})$ for $72 \mathrm{~h}$. In addition, the IC50 values of baicalein in DU145 and PC-3 cells were between 20 and $40 \mu \mathrm{mol} / 1$. We then selected the baicalein concentrations of 20 and $40 \mu \mathrm{mol} / 1$ for the subsequent experiments.

\section{Baicalein induces apoptosis in prostate cancer cells}

To investigate the effect of baicalein on apoptosis in $\mathrm{PCa}$ cells, staining with Annexin V-conjugated Alexa Fluor 488 and propidium iodide was used to analyze the percentage of apoptotic cells induced by baicalein. The lower right (LR) and upper right (UR) quadrants of the histograms showed the percentages of early and late apoptotic cells, respectively (Fig. 2a, b). The total percentage of apoptotic cells (UR + LR) increased from $13 \%$ in control DU145 cells to 23.75 and $36.65 \%$ in the cells treated with baicalein (20 and $40 \mu \mathrm{M}$, respectively) for $48 \mathrm{~h}\left({ }^{*} P<0.05\right.$, $* * P<0.01$ ) (Fig. 2c). A similar phenomenon was also found in the PC-3 cells, and the total percentage of apoptotic cells was increased from 13.82 to $17.17 \%$ and $29.38 \%$, respectively $(* P<0.05, * * P<0.01$ ) (Fig. $2 \mathrm{~d}$ ). The treatment of DU145 and PC-3 cells with 20 and $40 \mu \mathrm{M}$ baicalein for $48 \mathrm{~h}$ induced apoptosis in both cell lines in a dose-dependent manner, indicating the anti-tumor effect of baicalein on PCa cells.

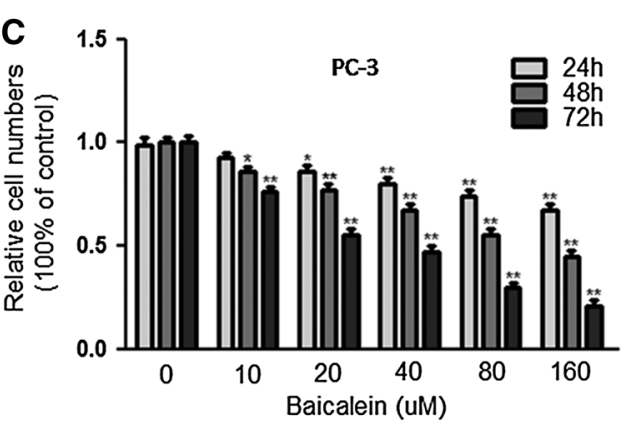

Baicalein significantly inhibited the viability of both cell lines in a dose-and time-dependent manner. The results are presented as the means \pm SD of three independent experiments and the corresponding standard error. $* P<0.05 ; * * P<0.01$ 
Fig. 2 Baicalein induces dosedependent apoptosis in DU145 and PC-3 cells. a DU145 and b PC-3 cells were treated with baicalein $(0,20$, and $40 \mu \mathrm{M})$ for $24 \mathrm{~h}$ and stained with FITCAnnexin V and PI. The percentage of surviving cells is shown in the lower left quadrant; and the percentages of early-stage and late-stage apoptotic cells are shown in the lower right and upper right quadrants, respectively. c, d The apoptosis induced by baicalein was quantified. The Data are presented as the means \pm SD of three independent experiments. $* P<0.05 ; * * P<0.01$

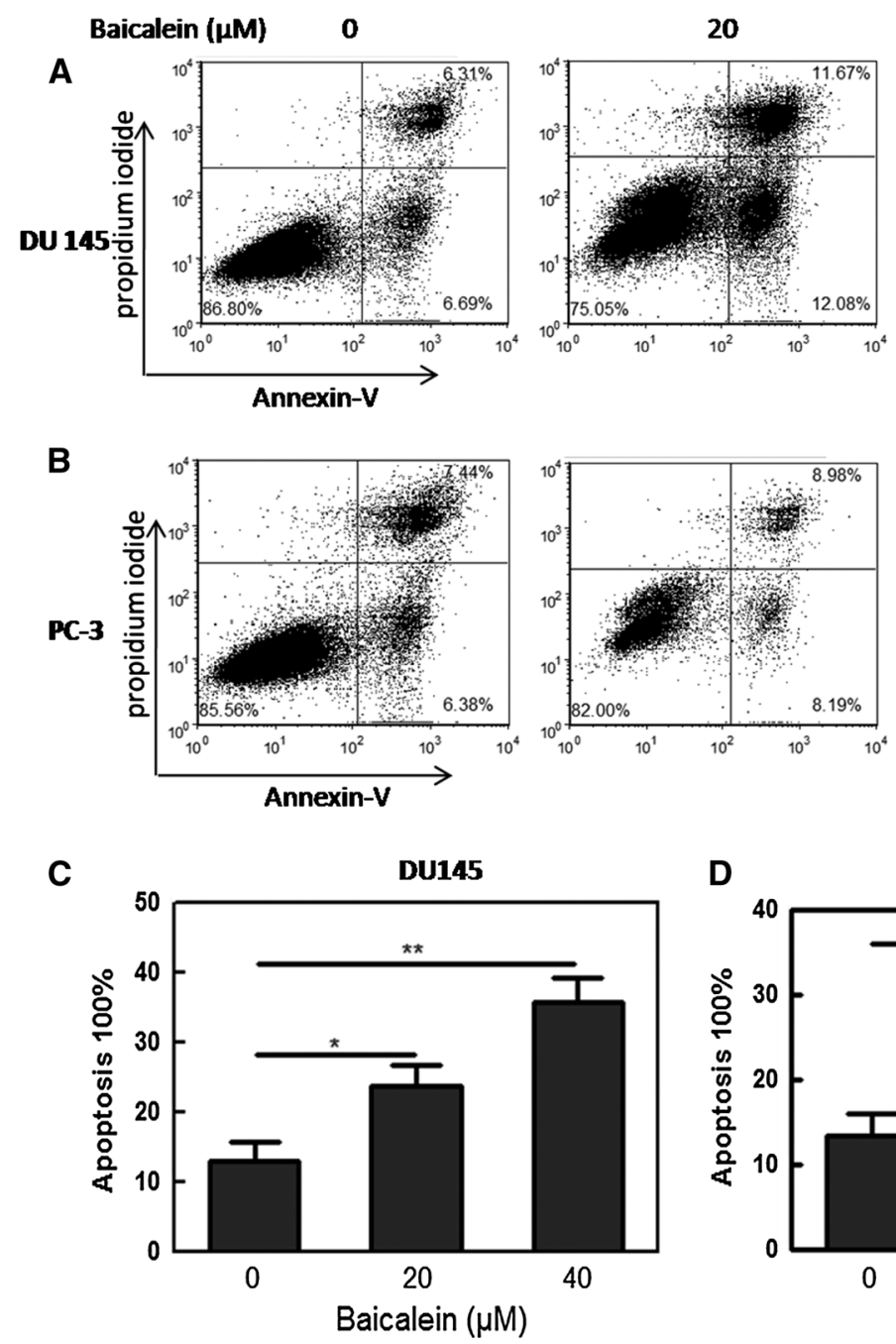

20
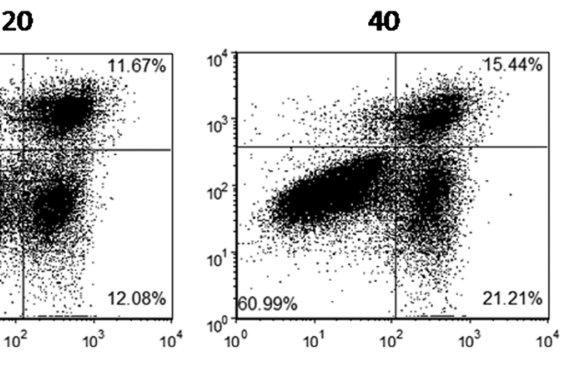

B

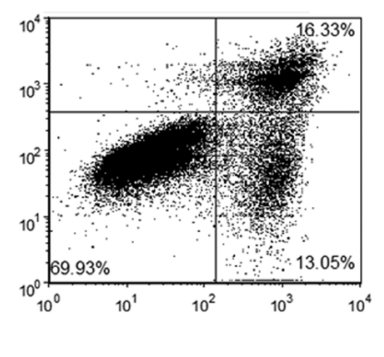

D

PC-3

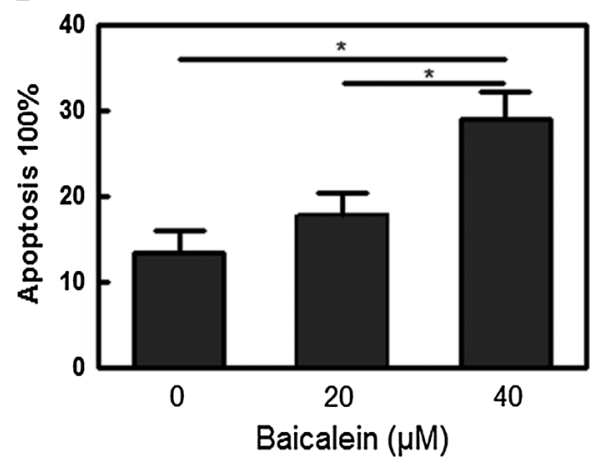

\section{Migration and invasion are inhibited by baicalein in prostate cancer cells}

The scratch assay was implemented to investigate the effect of baicalein on the migration of prostate cancer cells. As shown in Fig. 3a, b, the migration of DU145 cells was restrained by baicalein in a dose-dependent manner, and a similar effect was also observed in PC-3 cells (Fig. 3c, d). A transwell assay was then performed to further test the influence of baicalein on cell migration and invasion. Our results showed that baicalein can significantly inhibit DU145 cells migration and invasion $(* P<0.05, * * P<0.01)$ (Fig. 4a, b) in a dose-dependent manner. Furthermore, we observed the same potent effect of baicalein on PC-3 cells ( ${ }^{*} P<0.05$, $* * P<0.01$ ) (Fig. 4c, d).

\section{Effects of baicalein on the expression of cell apoptosis-related proteins}

It is well known that the expression of the pro-apoptotic protein Bax is associated with the increased apoptosis, whereas the anti-apoptotic protein Bcl-2 is associated with the inhibition of apoptosis in target cells [13, 14]. In addition, the $\mathrm{Bax} / \mathrm{Bcl}-2$ ratio plays a role in the regulation of apoptosis. As shown in Fig. 5a, the level of Bax was elevated, whereas the level of Bcl-2 was decreased after treatment with baicalein. The comparison of the intensity of their bands revealed that the $\mathrm{Bax} / \mathrm{Bcl}-2$ ratio was increased after baicalein treatment in a dose-dependent manner (Fig. 5b). Additionally, the expression of survivin was decreased, whereas the level of cleaved PARP was elevated.

\section{Baicalein inhibits the proliferation and metastasis of PCa cells via the caveolin-1/AKT/mTOR pathway}

A Western blotting assay was performed to detect the underlying mechanism through which baicalein exerts its actions. Caveolin-1 (cav-1) has been reported to mediate survival and promote metastatic activities in prostate cancer cells [15]. We found that treatment with baicalein resulted in a dose- and time-dependent decrease in cav-1 expression in PCa cells (Fig. 5c, d). Previous studies have 
Fig. 3 The scratch assay shows the effect of baicalein on cell migration. a Scratch assay of and $40 \mu \mathrm{M}$ baicalein. b The inhibition of migration was transformed to the percentage of the initial distance between the two edges. The baicalein-treated DU145 cells showed a lower rate of wound closure than the control cells. c Scratch assay of PC-3 cells treated with 0,20 , and $40 \mu \mathrm{M}$ baicalein. $\mathbf{d}$ The baicalein-treated PC-3 cells closure than the control cells DU145 cells treated with 0,20 , showed a lower rate of wound
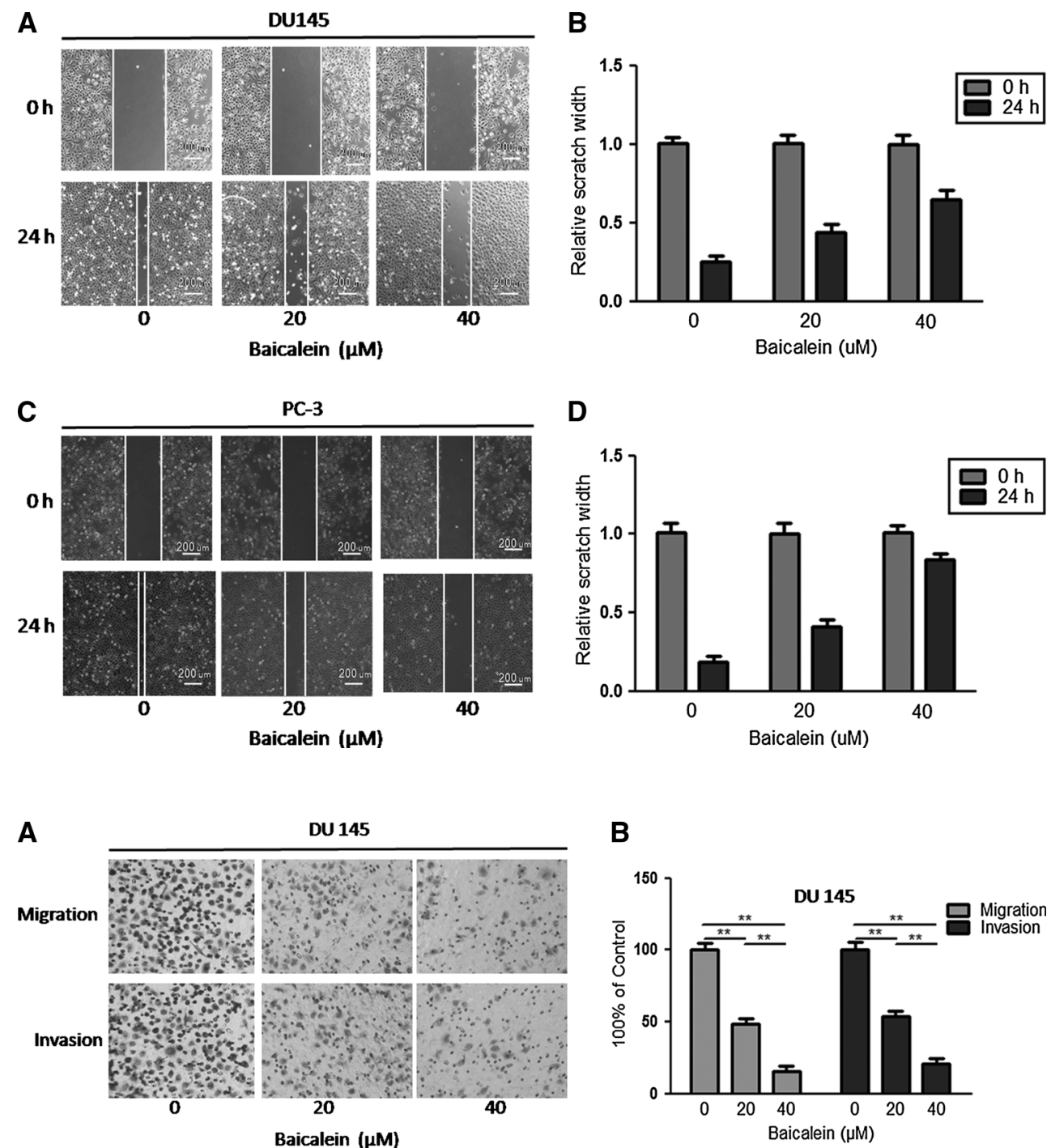

B

Fig. 4 Baicalein inhibits cell migration and invasion ability in a dose-dependent manner. a The migration and invasion of DU145 cells were inhibited after treatment with different concentrations of baicalein. b The number of DU145 cells that successfully migrated and invaded was counted.

c Treatment with 0,20 , and $40 \mu \mathrm{M}$ baicalein inhibited the migration and invasion of PC-3 cells. d The decrease in the number of PC- 3 cells indicates the marked inhibitory effect of baicalein on cell mobility. The Data are presented as the means \pm SD of three independent experiments. $* P<0.05 ; * * P<0.01$

\section{B}

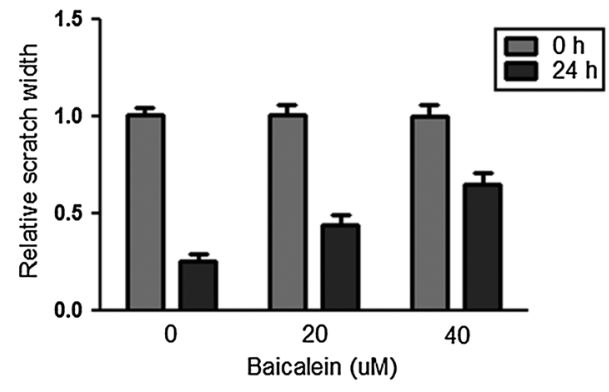

D

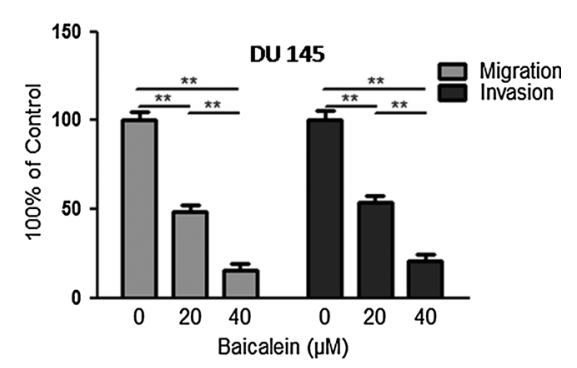

C

PC-3

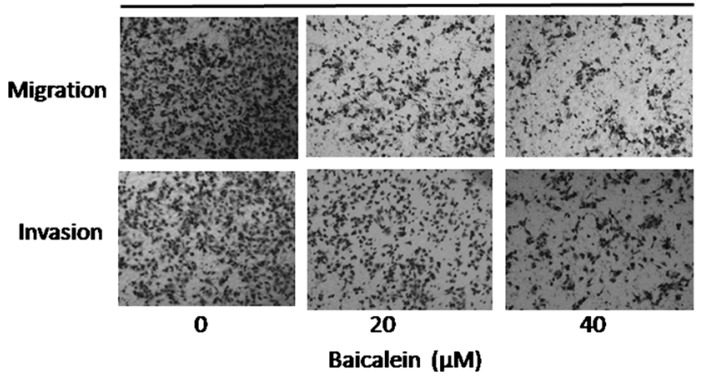

D

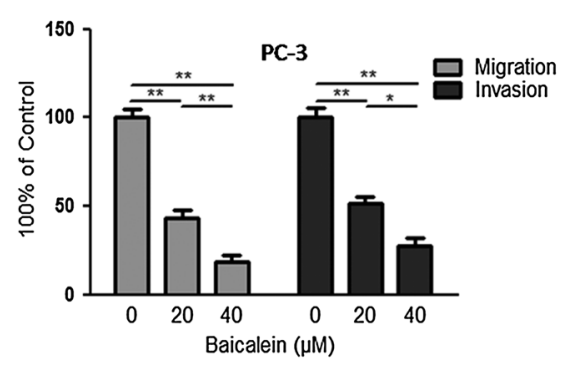

indicated that the PI3K/Akt/mTOR axis plays important roles in cancer cell proliferation, metabolism, migration, and angiogenesis [16-18], and that the inhibition of the AKT signaling pathway can induce apoptosis and prevent the invasion and metastasis of prostate cancer cells. Therefore, we tested whether baicalein affects the PI3 K/ Akt/mTOR pathway. The treatment of DU145 and PC-3 cells with baicalein $(20$ and $40 \mu \mathrm{M})$ for 24 and $48 \mathrm{~h}$ resulted in a marked downregulation of the phosphorylation levels of AKT and mTOR. In addition, the p-mTOR/mTOR and p-AKT/AKT ratios were also decreased in a dose- and time-dependent manner (Fig. 5e, f, g, h).

To investigate the role of AKT in the proliferation and mobility of PCa cells, DU145 and PC-3 cells were pretreated with a pan-PI3 K inhibitor (LY294002, $50 \mu \mathrm{M}$ ) for $30 \mathrm{~min}$ and then incubated in the presence or absence of baicalein $(20 \mu \mathrm{M})$ for $48 \mathrm{~h}$. The Western blotting analysis results indicated that the inhibition of AKT activity with 
Fig. 5 Effects of baicalein on the protein levels of Bax, Bcl-2, PARP, survivin, caveolin-1, AKT, and mTOR in DU145 and PC-3 cells. a Bax, Bcl-2, PARP, and survivin were assayed in DU145 and PC-3 cells by Western blotting analysis using GAPDH as a control. b Change in the $\mathrm{Bax} / \mathrm{Bcl}-2$ ratios in DU145 and PC-3 cells after treatment with baicalein. The densitometry value of each band was determined with ImageJ. The Data are presented as the means \pm SD of three independent experiments. $* P<0.05$; ** $P<0.01$. c-g The levels of caveolin-1, AKT, p-AKT, mTOR, and p-mTOR were analyzed by Western blotting. The p-AKT/ AKT and p-mTOR/mTOR ratios were quantified by densitometry analysis
A
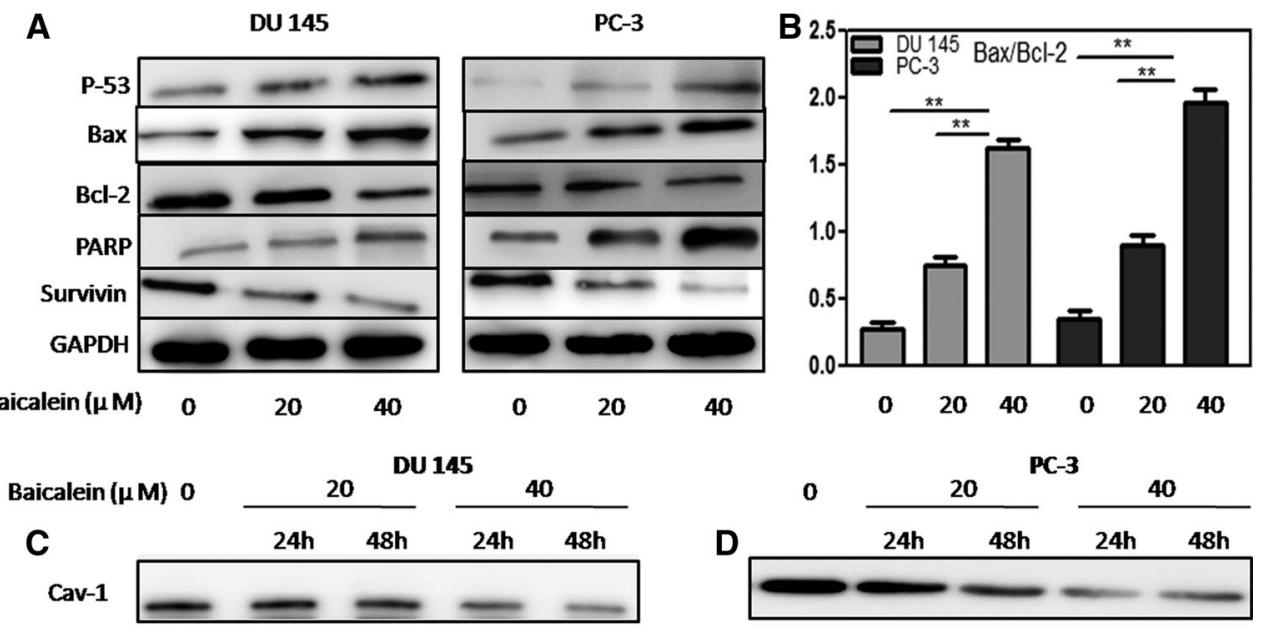

$E$

$\mathrm{E}_{\text {P-AKT }}$

AKT
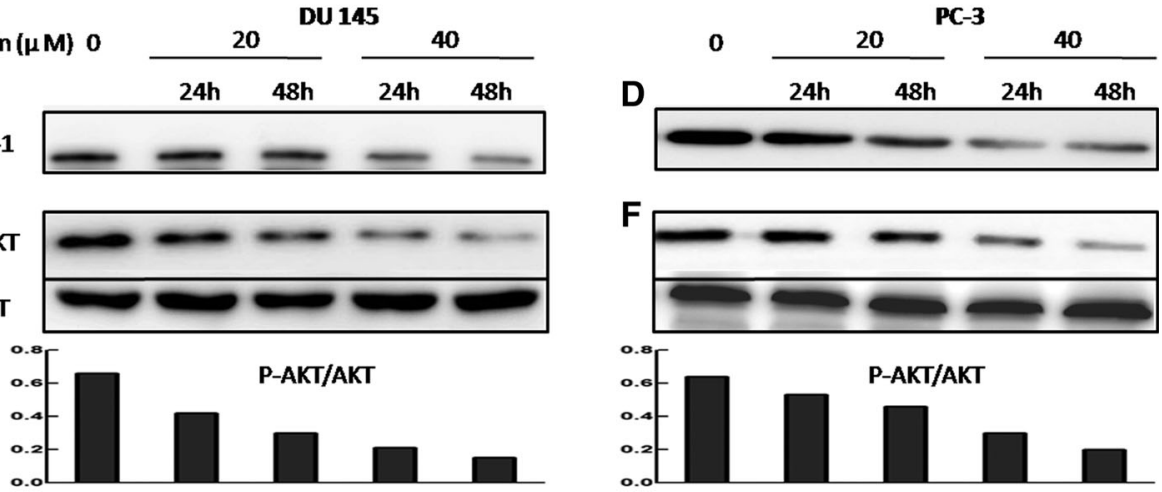

F
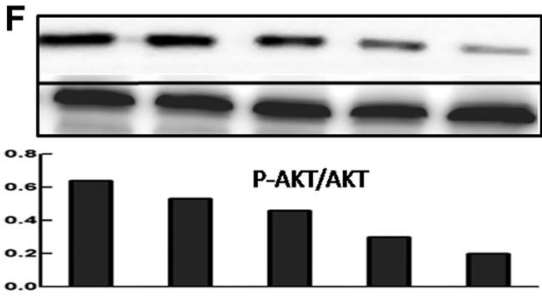

G
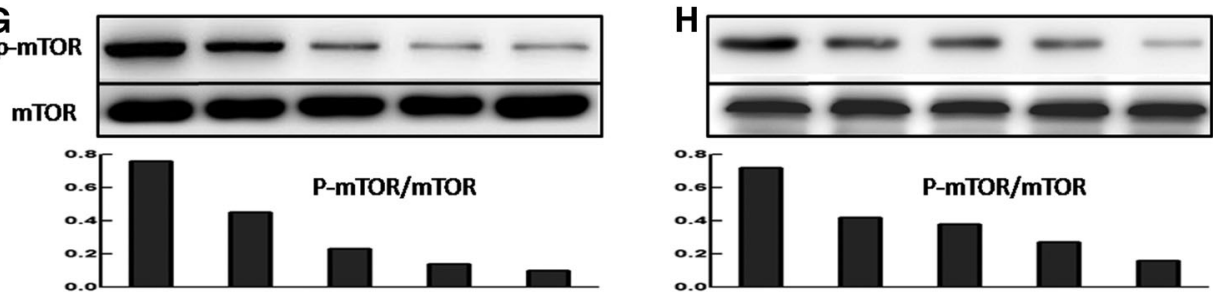

GAPDH
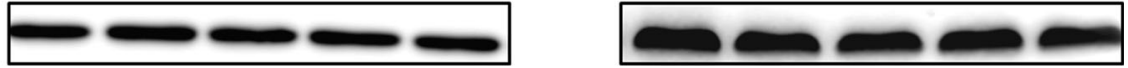

LY294002 could enhance the upregulation of Bax expression and downregulation of Bcl-2 expression induced by baicalein (Fig. 6a, b). We then evaluated the baicaleininduced apoptosis and anti-metastasis activity in PCa cells pretreated with LY294002. Consistently, the results showed that LY294002 significantly induced apoptosis (Fig. 6c, d) and suppressed cell invasion (Fig. 6e, f), indicating that $\mathrm{AKT}$ plays important roles in the apoptosis and invasion of PCa cells. In addition, the results revealed that LY294002 can markedly potentiate the pro-apoptotic and anti-metastasis effects of baicalein, which suggests that blocking the AKT signaling pathway may enhance the anticancer effect of baicalein (Fig. 7).

\section{Discussions}

Flavonoids are a subclass of polyphenolic compounds that have been widely used for thousands of years in Oriental medicine due to their anti-allergic, anti-inflammatory, and anti-microbial effects [19-21]. Recently, a series of studies have shown that flavonoids exhibit anti-tumor effects against various cancer cells of different origins [7, 22, 23]. The widespread distribution of flavonoids, their variety, and their relatively low toxicity compared with other active plant metabolites (e.g., alkaloids) had led to their consumption by human beings in significant quantities [24] Although low water solubility, fast oxidative degradation, and fast metabolism limit its pharmaceutical use in some degree, various methods have been used to overcome these issues of flavonoids [25]. The flavonoids family consists of various members, including flavones, flavonols, baicalensis, and anthocyanidins [26]. Of these drugs, the flavonoid baicalein has been found to promote the apoptosis of prostate cancer cells by regulating AR [27]. However, the effect of baicalein on apoptosis-related proteins in $\mathrm{PCa}$ cells and the effect of baicalein on the metastasis of $\mathrm{PCa}$ cells have not been elucidated.

In the present study, we demonstrated that baicalein can significantly inhibit the proliferation and induce apoptosis 

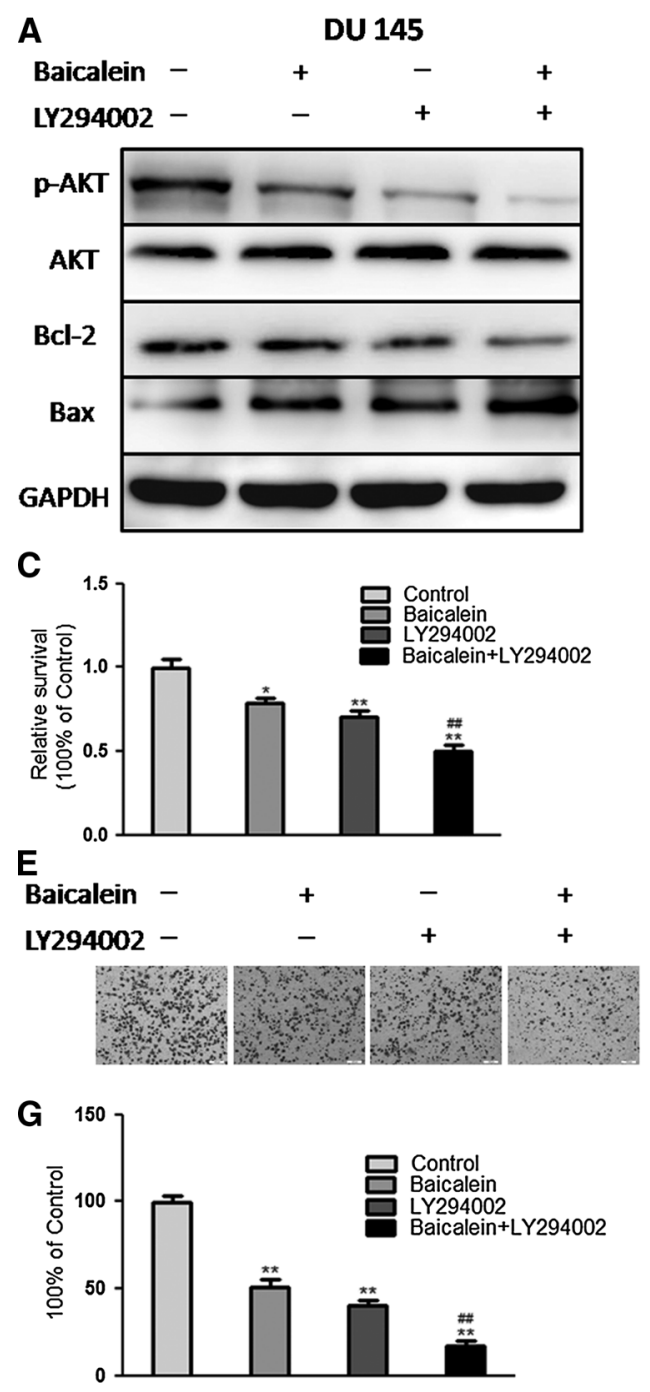

Fig. 6 Inhibition of AKT activation promotes apoptosis and inhibits the metastasis of PCa cells induced by baicalein. a, b Expression of p-AKT, AKT, Bax, and Bcl-2 in DU145 and PC-3 cells after treatment with baicalein and/or LY294002. After treatment with LY294002A, DU145 and PC-3 cells were incubated in the absence or presence of baicalein $(40 \mu \mathrm{M})$ for $48 \mathrm{~h}$. The cell viability was

of androgen-independent prostate cancer cells, in accordance with previous research findings [11]. We further explored the molecular mechanisms through which baicalein induces apoptosis. The results indicated that the regulation of cav-1 and apoptotic proteins is required. Our data indicate a new mechanism through which baicalein exhibits its pro-apoptotic effect in prostate cancer cells.

Cav-1 is a major structural component of the caveolae, which are specialized plasma membrane invaginations involved in molecular transport, cell adhesion, and signal transduction [28]. Cav-1 was recently found to function as an anti-apoptotic protein, which was associated with several members of the Bcl-2 family of proteins [29-31]. In the present study, we found that baicalein reduces a

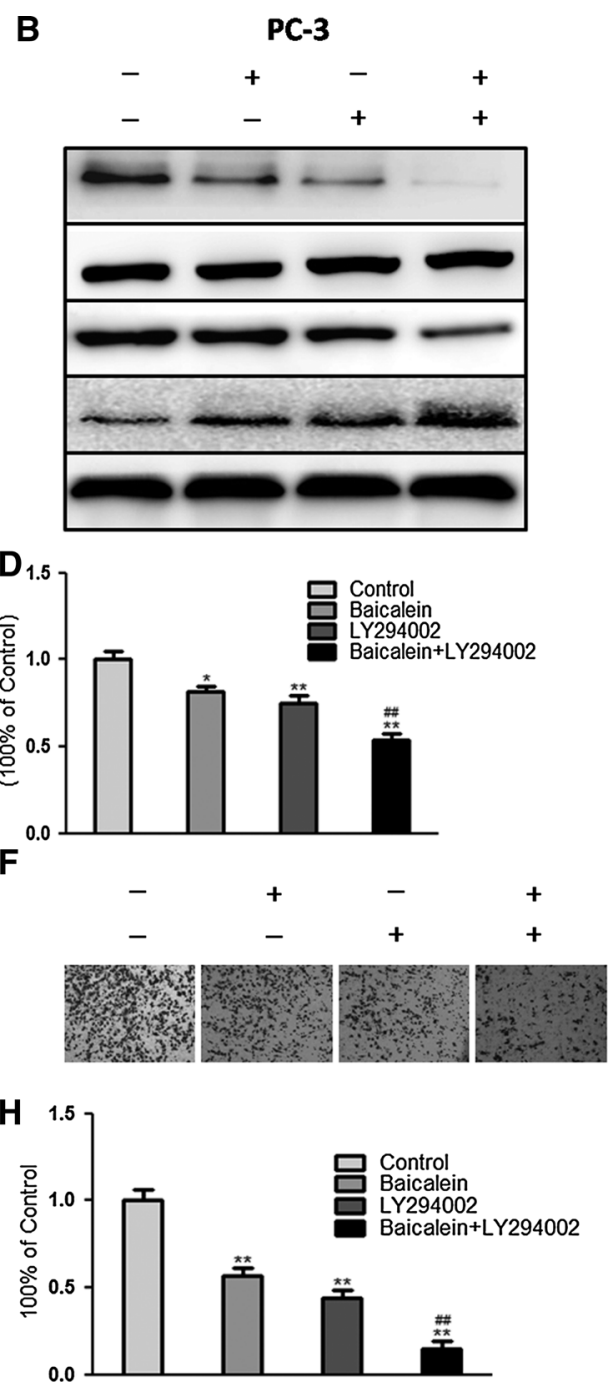

measured by MTT assay (c, d), and the cell invasion was measured by a Transwell assay $(\mathbf{e}, \mathbf{f})$. The results are presented as the means \pm SD of three independent experiments. $* P<0.05$; $* * P<0.01$, compared with the control cells. ${ }^{\#} P<0.05$ or ${ }^{\# \#} P<0.01$, compared with the cells treated with baicalein

time- and dose-dependent inhibition of cav-1, as demonstrated by a Western blot assay. Additionally, after exposure to different concentrations of baicalein, the proapoptotic Bax and PARP in DU145 and PC-3 cells were up-regulated, whereas the anti-apoptotic Bcl-2 and survivin were down-regulated in a dose-dependent manner.

The presence of metastasis is the main cause of mortality in patients with prostate cancer. Metastasis is a complex multistep process involving cell motility and invasion. Hence, the interruption of these steps is one approach for anti-metastatic therapy [32]. As shown through wound healing and Matrigel chamber invasion assays, baicalein markedly inhibited the migration and invasion of $\mathrm{PCa}$ cells in a concentration-dependent manner. Further 


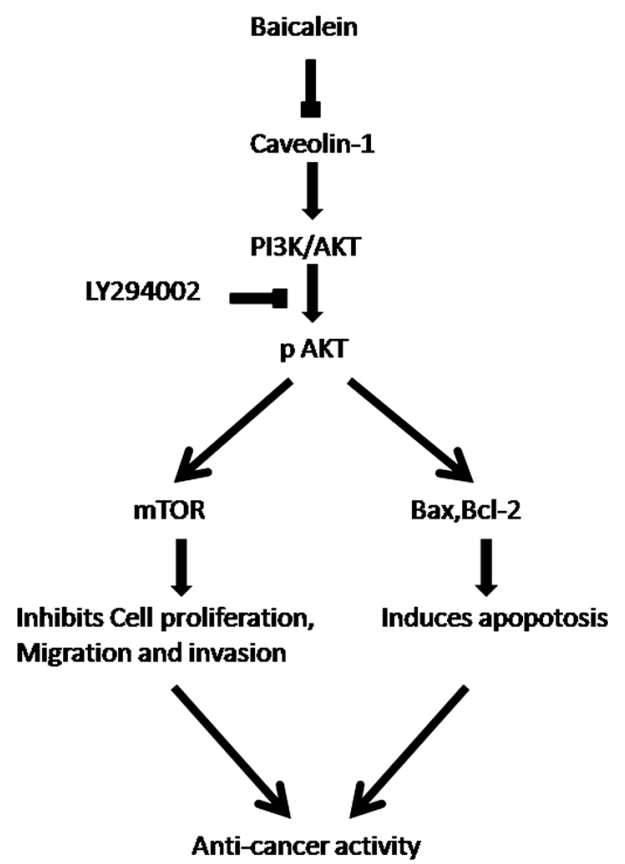

Fig. 7 Hypothesized model for the mechanism through which baicalein induces apoptosis and inhibits metastasis of prostate cancer cells. Exogenous baicalein promotes the apoptosis and inhibits the metastasis of androgen-independent prostate cancer by interrupting the caveolin-1/Akt/mTOR pathway

research of the molecular mechanisms indicated that the cav-1/AKT/mTOR signal pathway plays a vital role in this process.

With the exception of its effect on cell apoptosis, cav-1 was also reported to be associated with poor prognosis and metastasis in prostate cancer, and a reduction in cav-1 expression can decrease the tumorigenic and metastatic potential of prostate cancer, indicating that cav-1 is a potential target for prostate cancer [33-35]. PI3K/AKT is involved in a number of important cellular processes, including cellular survival and tumor metastasis [36]. Recent studies have indicated that the PI3K/Akt signaling pathway is overactive in a large range of cancers, and the inhibition of this pathway is considered a novel target for cancer therapy [37, 38]. Moreover, it has been reported that PI3K/ Akt exists in the caveola and is regulated by cav-1 $[39,40]$. In our study, we found that baicalein can significantly suppress the phosphorylation/activation of AKT in a timeand dose-dependent manner, in accordance with the inhibition of cav-1. To further investigate whether the proapoptotic and anti-metastatic effects are mediated by the cav-1/AKT pathway, LY294002, a PI3K inhibitor, was used to knockdown the expression of AKT. We found that LY294002 could markedly increase the baicalein-induced anti-cancer effect. In addition, it has been reported that AKT exerts its biological effect by phosphorylating its downstream substrates, including mTOR. Phosphorylated
mTOR can promote the proliferation, motility, and survival of cancer cells [41]. The results indicated that baicalein treatment also inhibits the mTOR phosphorylation and AKT activity. Thus, these data suggest that baicalein may inhibit the cav-1/AKT/mTOR pathway to induce its anticancer effect.

Additionally, Akt is reported to promote cell survival through the direct regulation of Bcl-2 family members [ 42 , 43]. Our data also demonstrated that the inhibition of AKT activity can enhance the upregulation of Bax expression and downregulation of Bcl-2 expression induced by baicalein, suggesting that AKT may be at the upstream of the baicalein-induced apoptosis of prostate cancer cells.

Taken altogether, our study demonstrates that baicalein markedly induces apoptosis and inhibits metastasis of androgen-independent prostate cancer cells. Mechanically, we find that cav-1/AKT/mTOR pathways account for the anti-tumor effects of baicalein. All these results imply that baicalein may be a promising therapeutic drug for androgen-independent prostate cancer patients.

Acknowledgments This work was supported by the National Natural Science Foundation of China (No. 81172435).

Open Access This article is distributed under the terms of the Creative Commons Attribution 4.0 International License (http:// creativecommons.org/licenses/by/4.0/), which permits unrestricted use, distribution, and reproduction in any medium, provided you give appropriate credit to the original author(s) and the source, provide a link to the Creative Commons license, and indicate if changes were made.

\section{References}

1. Jemal A, Siegel R, Ward E, Hao Y, Xu J, Thun MJ (2009) Cancer statistics, 2009. CA 59:225-249

2. Mohler JL, Gregory CW, Ford OH 3rd et al (2004) The androgen axis in recurrent prostate cancer. Clin Cancer Res 10:440-448

3. Agoulnik IU, Weigel NL (2006) Androgen receptor action in hormone-dependent and recurrent prostate cancer. J Cell Biochem 99:362-372

4. Hui C, Qi X, Qianyong Z, Xiaoli P, Jundong Z, Mantian M (2013) Flavonoids, flavonoid subclasses and breast cancer risk: a meta-analysis of epidemiologic studies. PLoS One 8:e54318

5. Geybels MS, Verhage BA, Arts IC, van Schooten FJ, Goldbohm RA, van den Brandt PA (2013) Dietary flavonoid intake, black tea consumption, and risk of overall and advanced stage prostate cancer. Am J Epidemiol 177:1388-1398

6. Li-Weber M (2009) New therapeutic aspects of flavones: the anticancer properties of Scutellaria and its main active constituents wogonin, baicalein and baicalin. Cancer Treat Rev 35:57-68

7. Wang L, Ling Y, Chen Y et al (2010) Flavonoid baicalein suppresses adhesion, migration and invasion of MDA-MB-231 human breast cancer cells. Cancer Lett 297:42-48

8. Chiu YW, Lin TH, Huang WS et al (2011) Baicalein inhibits the migration and invasive properties of human hepatoma cells. Toxicol Appl Pharmacol 255:316-326 
9. Wang AM, Ku HH, Liang YC, Chen YC, Hwu YM, Yeh TS (2009) The autonomous notch signal pathway is activated by baicalin and baicalein but is suppressed by niclosamide in K562 cells. J Cell Biochem 106:682-692

10. Huang WS, Kuo YH, Chin CC et al (2012) Proteomic analysis of the effects of baicalein on colorectal cancer cells. Proteomics 12:810-819

11. Bonham M, Posakony J, Coleman I, Montgomery B, Simon J, Nelson PS (2005) Characterization of chemical constituents in Scutellaria baicalensis with antiandrogenic and growth-inhibitory activities toward prostate carcinoma. Clin Cancer Res 11:3905-3914

12. Yan L, Xing Z, Guo $Z$ et al (2013) Somatostatin derivative (smsDX) targets cellular metabolism in prostate cancer cells after androgen deprivation therapy. PLoS One 8:e55790

13. Gavathiotis E, Suzuki M, Davis ML et al (2008) BAX activation is initiated at a novel interaction site. Nature 455:1076-1081

14. Pollack A, Cowen D, Troncoso P et al (2003) Molecular markers of outcome after radiotherapy in patients with prostate carcinoma: Ki-67, bcl-2, bax, and bcl-x. Cancer 97:1630-1638

15. Li L, Yang G, Ebara S et al (2001) Caveolin-1 mediates testosterone-stimulated survival/clonal growth and promotes metastatic activities in prostate cancer cells. Cancer Res 61:4386-4392

16. Courtney KD, Corcoran RB, Engelman JA (2013) The PI3K pathway as drug target in human cancer. J Clin Oncol 28:1075-1083

17. Liu P, Cheng H, Roberts TM, Zhao JJ (2009) Targeting the phosphoinositide 3-kinase pathway in cancer. Nat Rev Drug Discov 8:627-644

18. Hsieh AC, Liu Y, Edlind MP et al (2010) The translational landscape of mTOR signalling steers cancer initiation and metastasis. Nature 485:55-61

19. Sallusto F, Poupot R, Clergue M, DePalma R, Fournie JJ (2000) A flavonoid sulfate antigen activates human alphabeta CD8+ Th2 lymphocytes in pollen allergy. Eur J Immunol 30:964-968

20. Wall C, Lim R, Poljak M, Lappas M (2013) Dietary flavonoids as therapeutics for preterm birth: luteolin and kaempferol suppress inflammation in human gestational tissues in vitro. Oxid Med Cell Longev 2013:485201

21. Mahboubi M, Kazempour N, Boland Nazar AR (2013) Total phenolic, total flavonoids, antioxidant and antimicrobial activities of scrophularia striata boiss extracts. Jundishapur J Nat Pharm Prod 8:15-19

22. Belguise K, Guo S, Sonenshein GE (2007) Activation of FOXO3a by the green tea polyphenol epigallocatechin-3-gallate induces estrogen receptor alpha expression reversing invasive phenotype of breast cancer cells. Cancer Res 67:5763-5770

23. Hazgui S, Bonnomet A, Nawrocki-Raby B et al (2008) Epigallocatechin-3-gallate (EGCG) inhibits the migratory behavior of tumor bronchial epithelial cells. Respir Res 9:33

24. Bello IA, Ndukwe GI, Audu OT, Habila JD (2011) A bioactive flavonoid from Pavetta crassipes K. Schum. Org Med Chem Lett $1: 14$
25. Kim KH, Park YD, Park H et al (2014) Synthesis and biological evaluation of a novel baicalein glycoside as an anti-inflammatory agent. Eur J Pharmacol 744:147-156

26. Mansuri ML, Parihar P, Solanki I, Parihar MS (2014) Flavonoids in modulation of cell survival signalling pathways. Genes Nutr 9:400

27. Chen S, Ruan Q, Bedner E et al (2001) Effects of the flavonoid baicalin and its metabolite baicalein on androgen receptor expression, cell cycle progression and apoptosis of prostate cancer cell lines. Cell Prolif 34:293-304

28. Sternberg PW, Schmid SL (1999) Caveolin, cholesterol and Ras signalling. Nat Cell Biol 1:E35-E37

29. Linge A, Weinhold K, Blasche R, Kasper M, Barth K (2007) Downregulation of caveolin-1 affects bleomycin-induced growth arrest and cellular senescence in A549 cells. Int J Biochem Cell Biol 39:1964-1974

30. Yu Q (2006) Restoring p53-mediated apoptosis in cancer cells: new opportunities for cancer therapy. Drug Resist Updat 9:19-25

31. Zou H, Volonte D, Galbiati F (2012) Interaction of caveolin-1 with Ku70 inhibits bax-mediated apoptosis. PLoS One 7:e39379

32. Nam KS, Shon YH (2009) Suppression of metastasis of human breast cancer cells by chitosan oligosaccharides. J Microbiol Biotechnol 19:629-633

33. Williams TM, Lisanti MP (2005) Caveolin-1 in oncogenic transformation, cancer, and metastasis. Am J Physiol Cell Physiol 288:C494-C506

34. Iguchi K, Matsunaga S, Nakano T, Usui S, Hirano K (2006) Inhibition of caveolin-1 expression by incadronate in PC-3 prostate cells. Anticancer Res 26:2977-2981

35. Sugie S, Mukai S, Tsukino $H$ et al (2013) Increased plasma caveolin-1 levels are associated with progression of prostate cancer among Japanese men. Anticancer Res 33:1893-1897

36. Shack S, Wang XT, Kokkonen GC, Gorospe M, Longo DL, Holbrook NJ (2003) Caveolin-induced activation of the phosphatidylinositol 3-kinase/Akt pathway increases arsenite cytotoxicity. Mol Cell Biol 23:2407-2414

37. Morgensztern D, McLeod HL (2005) PI3K/Akt/mTOR pathway as a target for cancer therapy. Anticancer Drugs 16:797-803

38. Yap TA, Garrett MD, Walton MI, Raynaud F, de Bono JS, Workman P (2008) Targeting the PI3K-AKT-mTOR pathway: progress, pitfalls, and promises. Curr Opin Pharmacol 8:393-412

39. Anderson RG (1993) Caveolae: where incoming and outgoing messengers meet. Proc Natl Acad Sci USA 90:10909-10913

40. Kim HA, Kim KH, Lee RA (2006) Expression of caveolin-1 is correlated with Akt-1 in colorectal cancer tissues. Exp Mol Pathol 80:165-170

41. Kim JK, Jung Y, Wang J et al (2013) TBK1 regulates prostate cancer dormancy through mTOR inhibition. Neoplasia 15:1064-1074

42. Downward J (2004) PI 3-kinase, Akt and cell survival. Semin Cell Dev Biol 15:177-182

43. Maddika S, Ande SR, Panigrahi S et al (2007) Cell survival, cell death and cell cycle pathways are interconnected: implications for cancer therapy. Drug Resist Updates 10:13-29 